\title{
Adaptive modulation and coding selection mechanisms for single-cell LTE broadcasting in enterprise network
}

\author{
Mohammed Algharem* and \\ Mohd. Hasbullah Omar \\ InterNetWorks Research Group, \\ School of Computing, \\ UUM, Malaysia \\ Email: algharem@internetworks.my \\ Email: mhomar@uum.edu.my \\ *Corresponding author

\section{Deris Stiawan}

College of Computer Science and IT, Sriwijaya University, Indonesia

Email: deris@unsri.ac.id

\section{Ismat Aldmour and Rahmat Budiarto}

College of Computer Science and IT,

Al Baha University, Saudi Arabia

Email: iaaldmour@bu.edu.sa

Email: rahmat@bu.edu.sa

\begin{abstract}
LTE broadcast or evolved multimedia broadcast multicast service (eMBMS) enables operators to efficiently launch media services over LTE to meet mobile users' demand on multimedia services. This paper introduces two mechanisms that use the standard deviation (StD) of eMBMS user's modulation and coding scheme (MCS) level group operated on single cell mode to select its optimal MCS level efficiently. Typically, MCS level is selected on the basis of users' worst channel condition, which results in wasting network resources. Since the eMBMS performance directly depends on the selected MCS level, an efficient adaptive modulation and coding (AMC) scheme is essentially needed. The proposed mechanisms are simulated using LTE simulator, and the results show that the proposed mechanisms increase the eMBMS performance in terms of throughput, delay and packet loss ratio, compared to the worst channel gain and averaging mechanisms.
\end{abstract}

Keywords: enterprise networks; LTE; evolved Multimedia Broadcast Multicast Service; eMBMS; modulation and coding scheme; MCS; spectrum efficiency; adaptive modulation and coding; AMC. 
Reference to this paper should be made as follows: Algharem, M., Omar, M.H., Stiawan, D., Aldmour, I. and Budiarto, R. (2018) 'Adaptive modulation and coding selection mechanisms for single-cell LTE broadcasting in enterprise network', Int. J. Digital Enterprise Technology, Vol. 1, Nos. 1/2, pp.60-78.

Biographical notes: Mohammed Algharem received his BS degree in Computer Science from the University of Sanaa, Yemen in 2004. He received his MS degree in Computer Network from the University Putra Malaysia (UPM) in 2010. He is currently working towards his PhD degree in 4G Wireless Network at the Utara University Malaysia (UUM). His research interests include multimedia networking over $4 \mathrm{G}$ network, network simulation architectures, and wireless scheduling algorithm.

Mohd. Hasbullah Omar received his BEng degree in Electronics, Telecommunication and Computer Engineering from the University of Bradford, England in 1999. He then obtained his MSc in Information Technology and PhD in Information Technology from the Universiti Utara Malaysia in 2004 and 2012 respectively. His research interest includes wireless communication, communication system and computer networks. He is with Universiti Utara Malaysia since 1999 and currently holds a Senior Lecturer post since 2011 .

Deris Stiawan received his $\mathrm{PhD}$ degree in Computer Science, majoring in Computer Security from the Universiti Teknologi Malaysia in 2014. $\mathrm{He}$ is an Associate Professor as well as a member of the Computer Network and Information Security (COMNETS) research group at the Computer Engineering Department, Faculty of Computer Science, Universitas Sriwijaya, Indonesia. His current work is in computer and network security field, focusing on network attack characterisation and visualisation, and intrusion prevention/detection system. He has been a member of IEEE since $2010 . \mathrm{He}$ holds a Certified Ethical Hacker (CEH) and Certified Hacker Forensic Investigator (CHFI) licenses from EC-Council since 2011.

Ismat Aldmour is currently in Albaha University in Saudi-Arabia in its Computer Engineering and Science Department. He obtained his $\mathrm{PhD}$ from the University of Glamorgan (currently University of South Wales), Wales, UK, in the area of mobile wireless communications systems in 2008. His MSc and BSc were from the University of Jordan, Jordan in the field of electrical engineering/communications. He assumed a number of non-academic positions including a Research Engineer/Head of Electrical Testing at the Royal Scientific Society of Jordan. His current research interests include resource management in future $5 \mathrm{G}$ networks, IoT, WSNs and engineering education.

Rahmat Budiarto received his BSc degree from the Bandung Institute of Technology in 1986, MEng, and DrEng in Computer Science from the Nagoya Institute of Technology in 1995 and 1998 respectively. Currently, he is a Professor at the College of Computer Science and IT, Albaha University, Saudi Arabia. His research interests include IPv6, network security, wireless sensor networks and smart networks. 


\section{Introduction}

Mobile broadband users are demanding spontaneous access to video content, higher-quality experience, and more convergent mobile services than ever before. Owing to the popularity and adoption of smartphones and tablets, mobile subscriptions for high data consumption devices are expected to reach 6.5 billion by 2018 . Mobile data traffic is expected to grow 15 fold by the end of 2017, driven mainly by video. LTE Broadcast enables operators to efficiently launch media services over LTE to meet this demand. According to a report from Global-mobile Suppliers Association, as of April 2016, 494 LTE networks commercially launched in 162 countries. Telecom carriers across the world are in the middle of replacing their $2 \mathrm{G} / 3 \mathrm{G}$ technology with the LTE technology. This transition is almost complete in major markets such as the US, Korea, and China. In the US, about $79 \%$ of total data traffic on Verizon (VZ) is carried on its LTE network. Telstra has announced its 2G network switch-off from 2016 and all Singapore carriers from 2017. These facts confirmed the adoption of broadcast LTE technologies (Heyn et al., 2016; Anis et al., 2016; 3GPP, 2015).

Multimedia application becomes a dominant application/service in future networks, especially in cloud computing environment as well as enterprise network (Alghamdi et al., 2016). Anis et al. (2015) reports the results of the project Integration of Broadcast and Broadband in LTE/5G (IMB5). The IMB5 project explores the LTE-broadcast mode or evolved Multimedia Broadcast Multicast Service (eMBMS) within two detailed single-frequency-network (SFN) field trial networks in Erlangen and Munich, Germany and identifies potential improvements of the current broadcasting feature within LTE towards future releases of $4 \mathrm{G}$ and $5 \mathrm{G}$. Two different LTE software-defined-radio platforms (one based on the Open Air Interface framework and one from National Instruments) have been setup to support detailed field experiments with the existing and future extended eMBMS beyond the state-of-the-art in LTE release 12. On the application layer, it is shown in the project, that using LTE eMBMS, a flexible service mix of unicast mobile broadband and broadcast linear TV can be delivered. For country-wide deployment of eMBMS SFNs, physical layer waveform modifications like an increased cyclic prefix compared to the current LTE standard are recommended.

The eMBMS is considered the best choice when a number of subscribers request the same data at the same time (Monserrat et al., 2012; Lecompte and Gabin, 2012). eMBMS should support users with high throughput. Radio resource allocation in eMBMS is the most challenging problem as stated in Alexiou et al. (2010). According to Rong et al. (2008), Sheng et al. (2008) and 3GPP (2006), eMBMS spectrum efficiency (SE) is directly connected with the selected modulation and coding scheme (MCS) level. Rong et al. (2008) state that SE is directly related to the eMBMS throughput. Thus, the MCS level should be selected carefully.

In unicast transmission mode, each user periodically or non-periodically sends its channel quality indicator (CQI) feedback to its base station, which is called evolved Node $\mathrm{B}$ (eNodeB). The eNodeB, in turn, dynamically adjusts the MCS level for that user which leads to high SE and increases the system performance. On the other hand, eMBMS users listen to the same channel with different channel conditions. Therefore, the selected MCS level should satisfy all users. However, if the MCS level was selected according to users suffering bad channel conditions, the channels will be more robust against error and failure on the account of lower bit rates. Consequently, the users with good channel 
conditions will sacrifice their opportunity to receive the data at high bit rates, which results in that the radio spectrum is inefficiently used (Wang et al., 2008; Won et al., 2009). Therefore, eMBMS faces a challenge in selecting a proper MCS level which will be suitable for all eMBMS users.

In this paper, effective MCS selection mechanisms are introduced to improve eMBMS performance. The proposed mechanisms take the users dispersion into account by using the standard deviation (StD) of users' MCS to pinpoint the users with abnormal MCS level and build algorithms accordingly.

\section{The proposed MCS selection mechanisms}

Two mechanisms are proposed in this paper. Both mechanisms are based on adaptive MCS. Both mechanisms, as well, use the StD of users' MCS levels as the basis for selecting proper MCS level. We start by discussing the fundamental concept of adaptive modulation and coding (AMC) and the mechanisms suggested in the literature to assign proper MCS level in eMBMS.

\subsection{Adaptive modulation and coding}

AMC is a key technique to achieve peak data rates in LTE networks (Ji et al., 2012; Fan et al., 2011) thereby increasing their throughput and improving SE (Chen et al., 2011). The AMC is responsible to provide the user's channel condition to the packet scheduler at the eNodeB, which in turn selects the most proper MCS level to that user. Each user performs signal to interference and noise ratio (SINR) measurement for its channel, then maps the obtained SINR value to the corresponding CQI value. After that, the user sends the CQI as a feedback message to its eNodeB, which will adapt the transmission rate according to the CQI feedback (Ji et al., 2012; 3GPP, 2013). LTE network defines 15 levels of CQI depending on the channel condition which start with the worst level $(\mathrm{CQI}=1)$ and end with the highest level $(\mathrm{CQI}=15)$ (see Table 1). Moreover, there are different types of modulation in LTE network such as quadrature phase shift keying (QPSK), 16 quadrature amplitude modulation (QAM), and 64QAM. Each modulation type has several coding rates, which composes the 15 different MCS levels. As shown in Figure 1, users who are located near the eNodeB receive the transmitted data at high bit rate using 64QAM, while cell edge users receive the transmitted data at low bit. This is in order to maintain the connection quality and link stability without the need to increase the transmission power (Fantacci et al., 2009; Cohen et al., 2010).

The AMC can be implemented easily and efficiently in point to point (PtP) transmission mode because MCS for any user can be accurately chosen according to user's CQI feedback report. However, in point to multi-point (PtM) transmission mode (such as eMBMS), a group of users listen to the same broadcast channel, and each user suffers from different channel propagation conditions. The eNodeB should adopt a proper MCS level which is suitable for all users in terms of throughput and robustness (Kim and Cho, 2005; Liang et al., 2011). The eMBMS's SE and throughput directly depend on the selected MCS. According to 3GPP (2006), in OFDMA-based multicast system, the selection of MCS is very important to improve the SE. 
Table 1 The CQI indices and their interpretations

\begin{tabular}{lccc}
\hline CQI index & Modulation & Code rate $\times 1,024$ & Efficiency bit $/ \mathrm{Hz}$ \\
\hline 1 & QPSK & 78 & 0.1523 \\
2 & QPSK & 120 & 0.2344 \\
3 & QPSK & 193 & 0.3770 \\
4 & QPSK & 308 & 0.6016 \\
5 & QPSK & 449 & 0.8770 \\
6 & QPSK & 602 & 1.1758 \\
7 & 16QAM & 378 & 1.4766 \\
8 & 16QAM & 490 & 1.9141 \\
9 & 16QAM & 616 & 2.4063 \\
10 & 64QAM & 466 & 2.7305 \\
11 & 64QAM & 567 & 3.3223 \\
12 & 64QAM & 666 & 3.9023 \\
13 & 64QAM & 772 & 4.5234 \\
14 & 64QAM & 873 & 5.1152 \\
15 & 64QAM & 948 & 5.5547 \\
\hline
\end{tabular}

Source: 3 GPP (2013)

Figure 1 Different 64QAM, 16QAM, and QPSK vs. distance from the cell centre (see online version for colours)

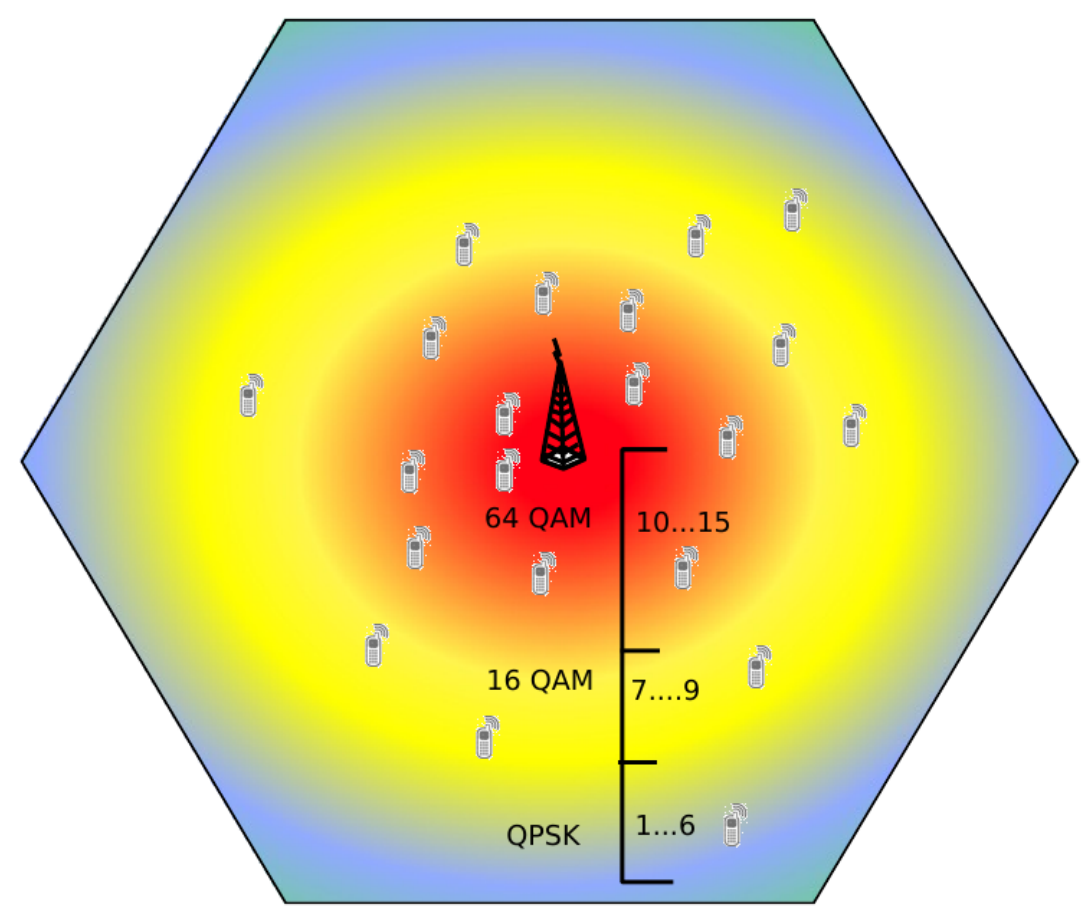


A user who is located close to its eNodeB can receive the signal at high data rate (e.g., 64QAM modulation level), whereas those who are located far from the eNodeB and suffer bad channel conditions can only reliably receive the signal at low data rates; using, say QPSK modulation. This is depicted in Figure 1. In the literature, there are several MCS selection mechanisms that have been proposed. We next review three of these mechanisms, namely; mechanisms based on fixed user's channel, average user's channel, and worst users channel conditions.

a Fixed predetermined MCS selection

A fixed MCS level will be selected to cover most of the eMBMS area (around 95\%). This mechanism promises to provide QoS with a high robustness and suppresses errors at the expense of throughput and SE (Kim and Cho, 2005). This is adequate if there are few users with steady transmission configurations and interference conditions (3GPP, 2006), otherwise it will waste the allocated bandwidth. The most advantage of fixed mechanism is that it does not need a feedback from eMBMS users.

b Worst user channel condition MCS selection

The easiest way to select an MCS level in PtM is to select the MCS level according to the worst case user among eMBMS users group. In the worst channel gain (WCG) mechanism, the lowest MCS of all users is selected. This mechanism causes a degradation in the system performance in terms of SE and throughput since the WCG does not take into account the users' distribution. The users who enjoy good channel conditions sacrifice their opportunity to receive the data at high bit rates (using high MCS levels). On the other hand, this mechanism provides the eMBMS service with high robustness, suppresses errors, and ensures fairness, regardless of the SE (Kim and Cho, 2005; Liang et al., 2011; Bochrini et al., 2013; Alexiou et al., 2010).

c Average MCS selection

In this mechanism, users frequently send their CQI feedback to their eNodeB which then maps the CQI to the corresponding average MCS level. The eNodeB calculates the average MCS level using equation (1) (Kim and Cho, 2005).

$$
\overline{M C S}=\frac{1}{N} \sum_{i=1}^{N} M C S_{i}
$$

where $M C S_{i}$ is the MCS level for user $i$ and $N$ is number of users who successfully sent CQI feedback.

This mechanism increases the throughput and the SE if the StD of MCSs is small. However, it cannot guarantee the QoS for all users, especially those who are suffering bad channel condition. The average MCS mechanism cannot satisfy all users' settings, especially when users' MCS levels are scattered. Thus, low MCS values of few users will slightly affect the average MCS value since there are users with high MCS values. Consequently, those users with low MCS level will suffer high bit error rate. The same mechanism was also proposed by others, e.g., in Won et al. (2009), Bochrini et al. (2013) and Koh and Kim (2006). We now proceed to present the two mechanisms proposed in this paper. 


\subsection{The proposed mechanisms}

Upper and lower thresholds are calculated in both proposed mechanisms in order to determine the users who have abnormal MCSs. The abnormal users are expensive to cover and cause eMBMS performance degradation. Having done mapping the received CQIs to the corresponding MCSs at the eNodeB, thresholds calculations are summarised in the following steps:

Step 1 Calculate the average of MCS value of all eMBMS users $N$, using equation (1).

Step 2 Calculate the StD $\sigma$ of MCSs values of all users using equation (2).

$$
\sigma=\sqrt{\frac{1}{N} \sum_{i=1}^{N}\left(M C S_{i}-\overline{M C S}\right)^{2}}
$$

Step 3 Calculate the upper threshold $T_{u p}$ and the lower threshold $T_{\text {low }}$ for all users using equation (3) and equation (4).

$$
\begin{aligned}
& T_{\text {up }}=\overline{M C S}+\sigma \\
& T_{\text {low }}=\overline{M C S}-\sigma
\end{aligned}
$$

$T_{u p}$ and $T_{l o w}$ values will be used to detect users with abnormal MCS level who are deviated away from the average of users' MCS average. All the above steps for the proposed mechanisms are explained in Algorithm 1.

Next we proceed to describing the two proposed mechanisms which both use the outputs of Algorithm 1; $T_{u p}$ and $T_{l o w}$ values.

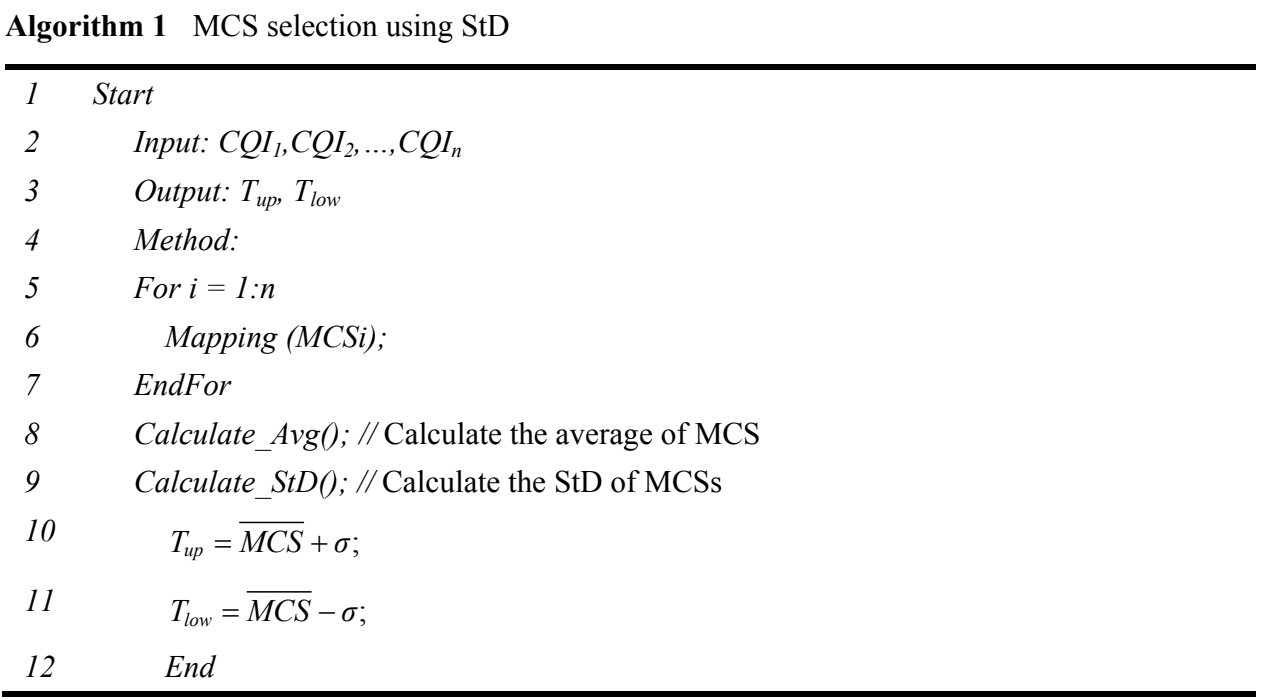

\subsubsection{The $M-W C G$}

The first proposed mechanism named as modified-worst channel gain (M-WCG). This mechanism uses the StD to exclude users with abnormal MCS level values. In a case where few users are located at the cell edge and the rest of the users are located close to 
eNodeB as shown in Figure 2, selecting the MCS corresponding to the WCG as proposed in previous works such as Kim and Cho (2005), Bochrini et al. (2013) and Alexiou et al. (2010) will result in wasting the spectrum and degrades eMBMS performance. In contrast, using the M-WCG mechanism to select a proper MCS, will exclude the users with abnormal MCS value from the worst user case calculation processes.

Figure 2 A cell with 18 users located at the cell centre and two users are located at the cell edge (see online version for colours)

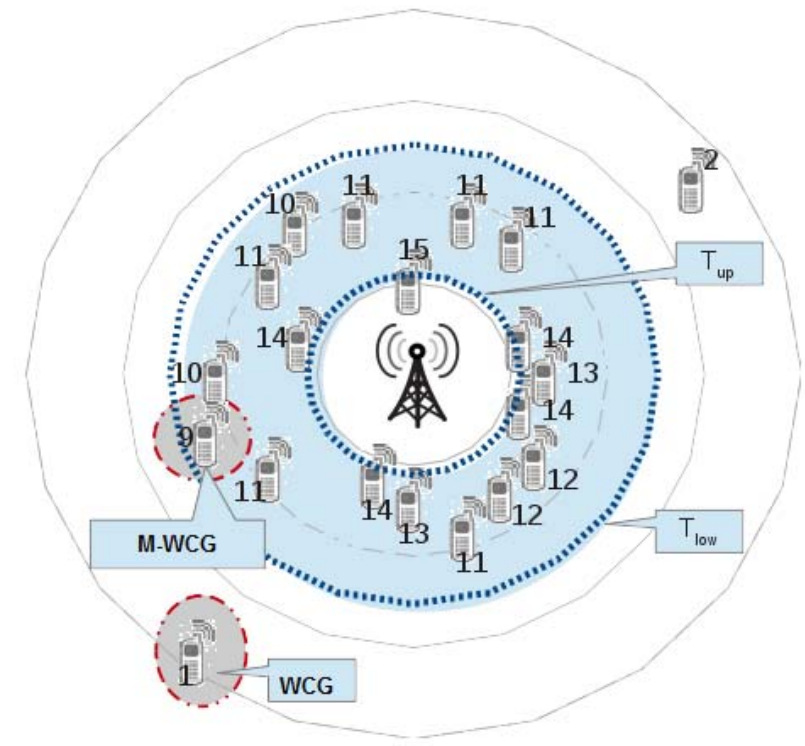

Transmitting the data to a multicast group according to the WCG mechanism will increase the fairness between users at the expense of system throughput degradation. The performance of the multicast network is bounded by the user in worst channel condition who acts as a bottleneck phenomenon (Chen et al., 2012). In 2009, Zhang et al. proposed a mechanism to overcome the worst user bottleneck by using a SINR threshold to sacrifice the users who are expensive to cover. Users with G-factor less than the threshold will be excluded from link adaptation and packet scheduling. The G-factor (called static SINR) is obtained by considering shadowing and large-scale attenuation without fast fading. In this research, the same mechanism will be used, but using different threshold, now based on StD of users' MCSs. The lower and upper thresholds will be used to classify the MCSs of each multicast group into two subgroups; normal subgroup and lower abnormal subgroup denoted by $M C S_{N G}$ and $M C S_{\text {lower }}$ respectively, where:

$$
\begin{aligned}
& M C S_{N G}^{v}=\left\{M C S_{i} \mid \forall M C S_{i} \geq T_{\text {low }}\right\} \\
& M C S_{\text {lower }}^{v}=\left\{M C S_{i} \mid \forall M C S_{i}<T_{\text {low }}\right\}
\end{aligned}
$$

The eNodeB will transmit the data with rate corresponding to the WCG in the $M C S_{N G}^{v}$, as in equation (7). Thus, users with MCS levels smaller than $T_{\text {low }}$ will receive the data with higher bit rate error compared to the users whose MCS in the normal subgroup. 


$$
M C S_{M_{-} W C G}=\operatorname{argmin}\left\{M C S_{N G}^{v}\right\}
$$

\subsubsection{The M-AVG MCS selection mechanism}

In the M-AVG, the $T_{u p}$ will be used to exclude the UEs with extremely high MCS. This exclusion can be achieved by excluding the MCSs which are larger than the $T_{u p}$, as used in equation (8) and equation (9).

$$
\begin{aligned}
& \overline{M C S}_{N G}=\text { average }\left\{M C S_{N G}^{v}\right\} \\
& M C S_{N G}^{v}=\left\{\forall M C S_{i} \leq T_{u p}\right\}
\end{aligned}
$$

Figure 3 The M-AVG MCS level selection at the cell edge (see online version for colours)

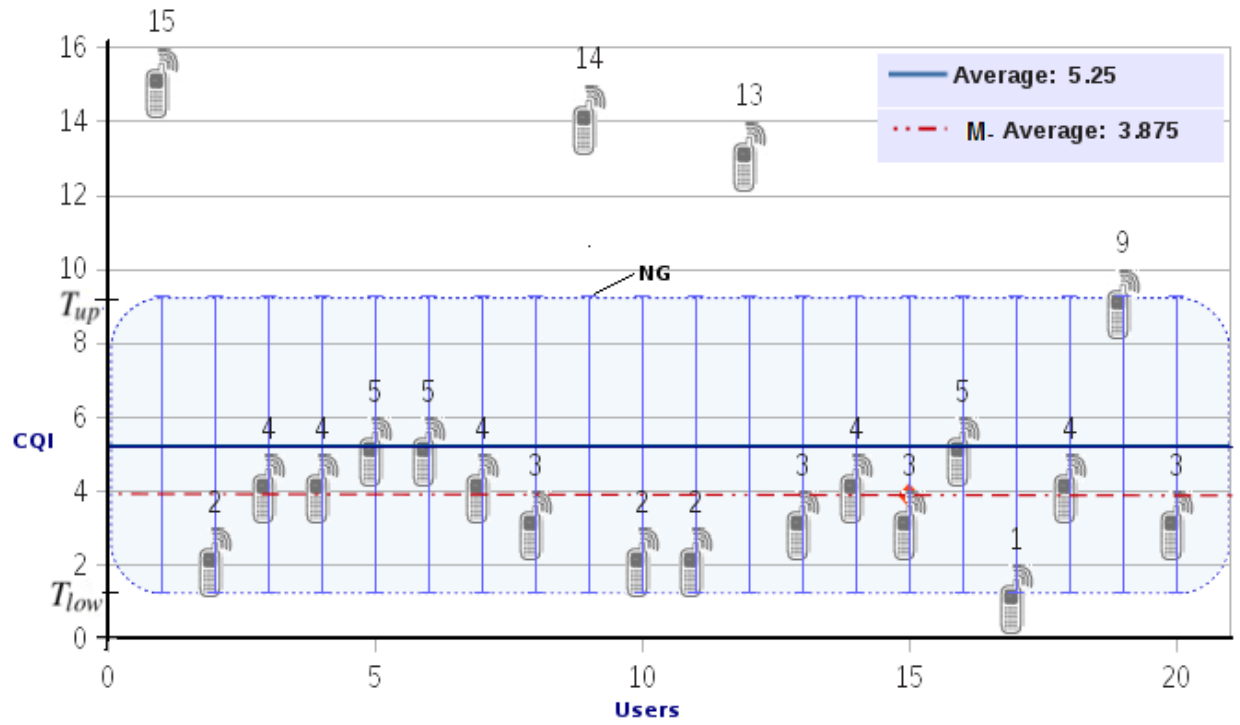

As illustrated in Figure 3, three abnormal users are excluded from the NG users because they have abnormal MCS levels compared to the rest of the users. The total MCS average for all users is 5.25 (the blue solid line), where the average of the NG users is 3.875 (the red dotted line). As it can be seen in the figure, the red dotted line is more close to users compared to the blue line. As a result, the M-AVG (red dotted line) is more suitable MCS level for eMBMS compared to the average of all MCSs.

To begin with, an experiment has been run on two of the mechanisms described above from literature (not the proposed ones). This is done in order to understand the effect of the selected MCS level on eMBMS performance. In the experiment, 15 scenarios with different MCSs $(0,2,4, \ldots, 28)$ were performed and repeated ten times. In each scenario, the MCS was selected at the beginning and kept throughout the simulation. For more fairness, the same random seed value has been used for each run. Moreover, 60 user equipment join the multicast session to receive the same video file. Other simulation parameters are listed in Table 2. 
Table 2 Simulation parameters

\begin{tabular}{lccc}
\hline Parameter & Value & Parameter & Value \\
\hline Carrier frequency & $2 \mathrm{GHz}$ & UE trans. pwr. & $23 \mathrm{dBm}$ \\
Path loss & $\begin{array}{c}\mathrm{PL}(\mathrm{db})=128.1+37.6 \\
\times \log \mathrm{d}, \mathrm{d} \mathrm{in} \mathrm{Km}\end{array}$ & Modulation schemes & QPSK, 16QAM, \\
Thermal noise & $-174 \mathrm{dBm} / \mathrm{Hz}$ & Number of eMBMS & groups \\
& & User distribution & Random and uniform \\
Downlink BW & $3 \mathrm{MHz}$ & User speed & $30 \mathrm{~km} / \mathrm{h}$ \\
Symbols for TTI & 12 & User mobility model & Random direction \\
Sub-frame length & $1 \mathrm{~ms}$ & Application flows & Video \\
Frame type & $\mathrm{FDD}$ & Simulation time(s) & 100 \\
eNodeB radius & $1 \mathrm{Km}$ & & \\
eNodeB pwr. trans. & $43 \mathrm{dBm}$ & & \\
\hline
\end{tabular}

Figure 4 The performance of eMBMS vs. different MCS level (see online version for colours)



The experiment was first done using the fixed MCS mechanism. The results are shown in Figure 4 in which the fairness index (FI), packet loss ratio (PLR) and normalised throughput for each scenario were plotted together. From the figure, we see that the FI remains at the maximum value until the selected MCS is eight. Then it starts to drop and continue dropping until it reaches its minimum value when as the MCS is 28 . This can be explained on the basis that for MCS values That are less or equal to eight, all UEs can receive the data, while some UEs cannot receive the transmitted data for MCS values greater than eight, and as the MCS level increases more, the number of those user equipment who cannot receive the data increases as well as which result in a more decrease in the FI. On other hands, the throughput is at its minimum value when the MCS equals to zero, then it starts to increase as the selected MCS increases. This continues until it reaches a maximum at MCS of 12 and it falls beyond that. The optimal FI (maximum value) and PLR (minimum value) combination occurred when the MCS is eight. The PLR parameter varies in an opposite way to the throughput variation. However, the throughput reaches its maximum value a while after the optimum FI/PLR 
combination, i.e., at a point ( $\mathrm{FI}=0.84, \mathrm{PLR}=0.26, \mathrm{MCS}=12)$ after that the FI starts to decrease and the PLR starts to increase. Hence, for maximum throughput, some sacrifice in fairness and extra packet losses has to be tolerated.

The same scenarios were performed using the WCG mechanism instead of the fixed MCS. In order to compare the performance of the fixed and WCG, the selected working point by the WCG is shown on the same graph in Figure 4. Clearly, the WCG selects an MCS level which reduces the eMBMS performance in terms of PLR and throughput. Meanwhile, there is a chance to select another MCS level which can improve the eMBMS performance with little impact on FI. From this point, the M-WCG was proposed in this paper to overcome the limitation of the WCG.

LTE simulator (LTE-Sim) has been used to evaluate the proposed mechanisms after extremely modifying and extending its functions and classes in order to support the eMBMS network. LTE-Sim is an open source framework simulator developed by Piro et al. (2011).

\subsection{Simulation scenarios}

Simulation experiments similar to those described in the previous section were applied in this section as well with more performance measures recorded. The M-WCG mechanism has been compared with the conventional WCG mechanism in terms of PLR, throughput, FI, and delay. Moreover, the M-AVG was compared with the conventional average mechanism. Only eMBMS has been activated in the simulation, so no unicast services have been used during the eMBMS session. The whole bandwidth was assigned to the eMBMS and shared between all sessions. We simulated all mechanisms through three scenarios. In first scenario we experimented using different number of multicast groups, each group has the same number of users ( $\mathrm{N}=60$ and video rate equal to $440 \mathrm{kbps})$. In the second scenario, we experiment using different video rates with single group of 60 users. Finally, a single group with different number of users has been evaluated using a video rate equal to $440 \mathrm{kbps}$ in the third scenario. Moreover, the network traffic was generated from a real video file with $440 \mathrm{kbps}$. In order to scale the $440 \mathrm{kbps}$ trace file to generate new trace files with different rates, we followed the two steps method as described in Seeling and Reisslein (2005). Since the video application is sensitive to delay, the maximum delay allowed was set to 0.06 second. The scheduler will drop any packet exceeding this. For more accurate results, each scenario was repeated ten times, and then the average performance values were calculated. The simulation parameters are listed in Table 2.

\section{Simulation results}

\subsection{Different groups number}

Figure 5 shows the PLR vs. number of eMBMS groups. The M-WCG mechanism provides lower PLR compared to the WCG. This is because WCG selects the lowest 
MCS level which results in low bit rate transmission, and this, in turn, forces the packets to wait in the MAC queue until they are transmitted or dropped once a packet delay exceeds the maximum allowable delay period. The same reason makes the delay of WCG bigger than the delay of the M-WCG, as shown in Figure 7. In fact, the WCG provides lower packet error rate when comparing to the M-WCG. However, when the number of sessions was increased, each session will get a lower bandwidth which may not be enough to transmit the data with low MCS level and then causes in high PLR at the transmitter. As a consequence of the low PLR and high bit rate, M-WCG make its throughput better than the throughput of the WCG mechanism, as plotted in Figure 6. These enhancements of the M-WCG are attained on account of FI degradation. This is shown in Figure 8 where the WCG provides a higher FI compare to the M-WCG. This is because M-WCG transmits the data at a little bit higher rates which cannot be decoded by all UEs.

Figure 5 PLR of M-WCG and WCG vs. number of multicast groups (see online version for colours)

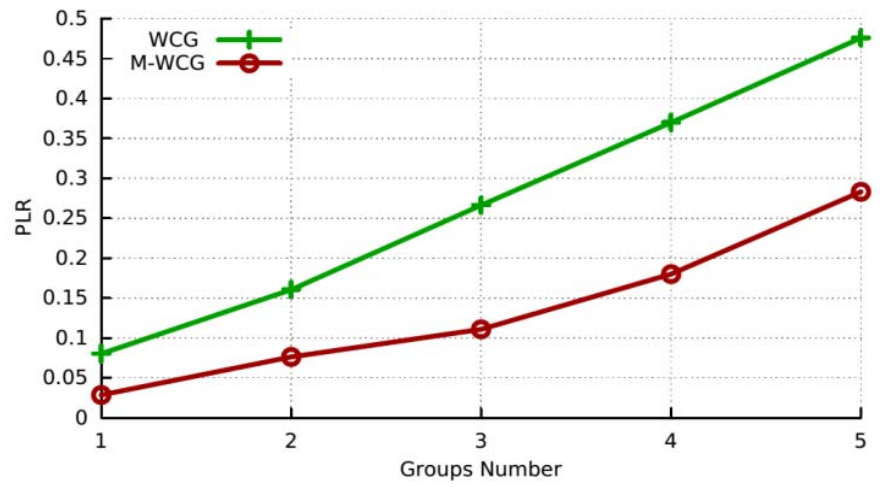

Figure 6 Throughput of M-WCG and WCG vs. the number of multicast groups (see online version for colours)

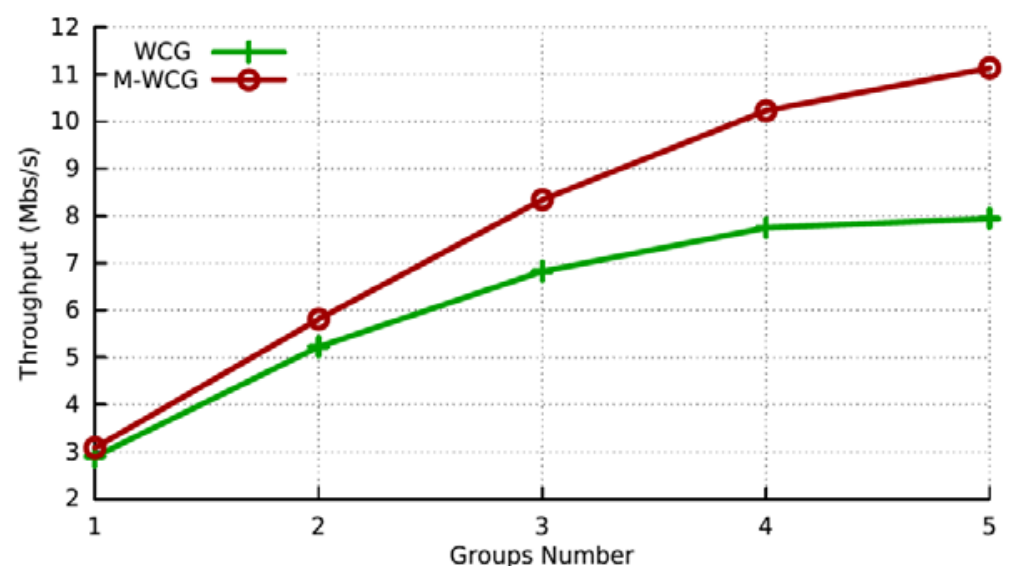


Figure 7 Delay of M-WCG and WCG vs. the number of multicast groups (see online version for colours)



Figure 8 FI of M-WCG and WCG vs. the number of multicast groups (see online version for colours)

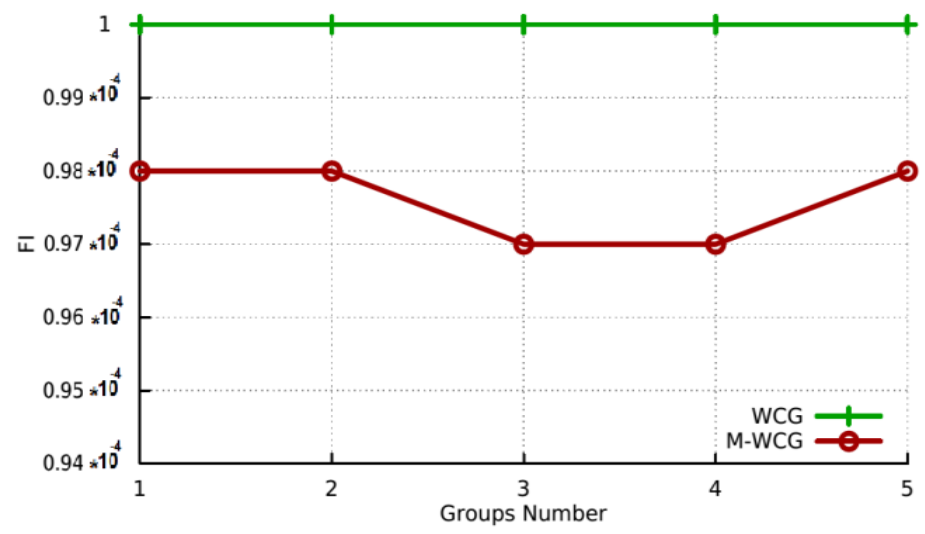

Figure 9 Throughput of M-AVG and average vs. number of multicast groups (see online version for colours)

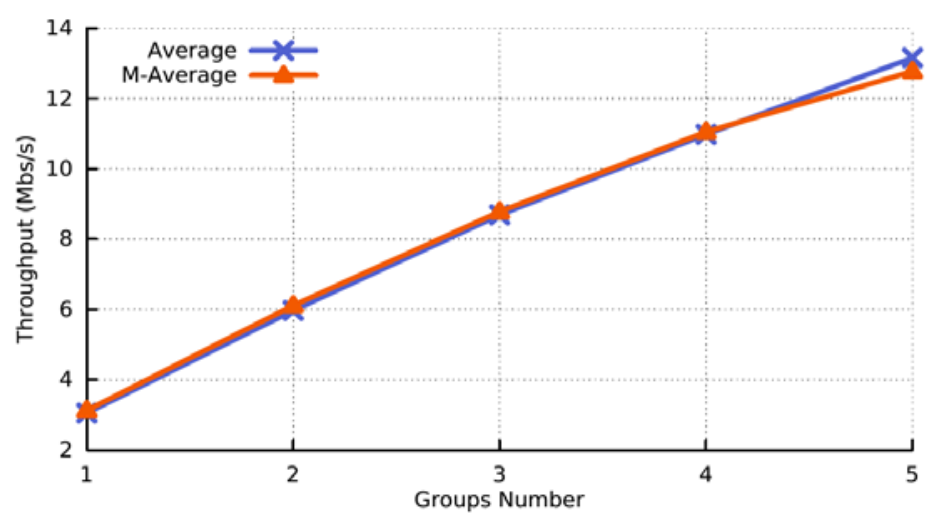


Figure 9 shows that the M-AVG MCS selection mechanism introduces higher throughputs than the conventional Average MCS selection mechanism. The M-AVG MCS selection mechanism provides low PLR compared to the conventional MCS average selection as exhibited in Figure 10. When the number of mobile groups increases, here exceeding four groups, the number of radio resources assigned to each group will not be enough to transmit the data using the selected MCS. Thus, the dropped packets at the MAC layer will increase, as well as, the waiting time of each packet to get scheduled and transmitted through the physical layer, as illustrated in Figure 11. Finally, the FI of all mechanisms are plotted in Figure 12. The best FI was introduced by M-WCG, WCG, and then M-AVG. whereas, the average mechanism introduces the lowest FI when comparing to other mechanisms.

Figure 10 PLR of M-AVG and average vs. number of multicast groups (see online version for colours)



Figure 11 End-to-end delay of M-AVG and average vs. the number of multicast groups (see online version for colours)

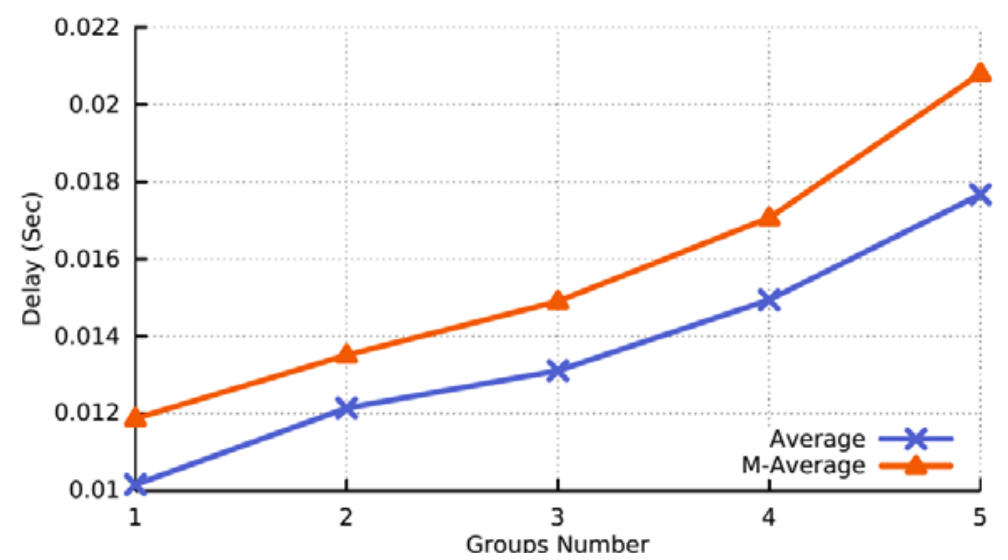


Figure 12 FI of M-AVG and average vs. the number of multicast groups (see online version for colours)

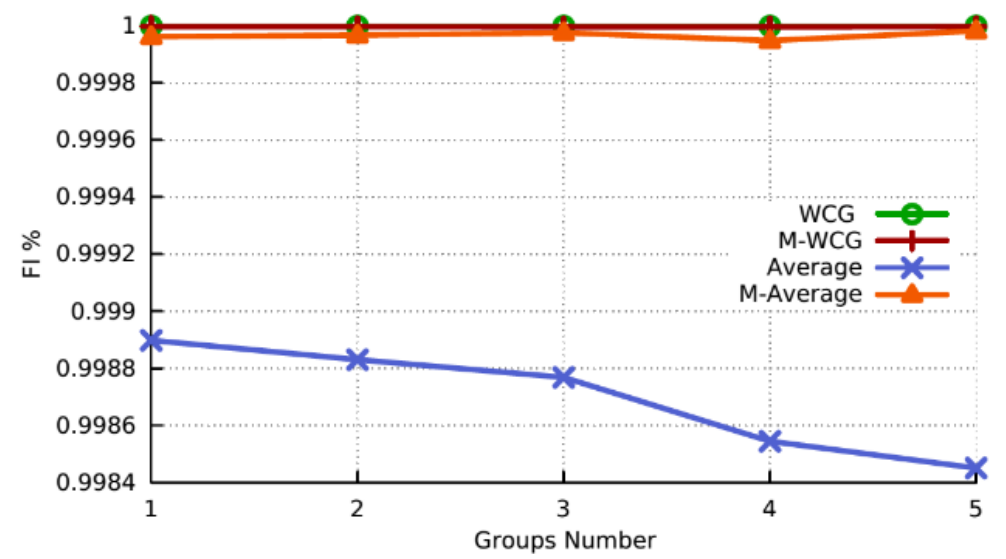

\subsection{Different video rates}

In this scenario, we evaluated both the proposed M-WCG and M-AVG mechanisms and the existing WCG and AVG mechanisms. As shown in Figure 13, throughput and delay of all mechanisms increase as the video rate expands, whereas, the block error ratio (BLER) and FI are slightly affected by expanding the video rate. However, the increase in throughput will continue until the transmitted video rate exceeds the available network resources.

As shown in Figure 13(a) at the low-rate video (such as $440 \mathrm{kbps}$ ), the proposed M-WCG mechanism produces better throughput than the other mechanisms, whereas, the Average mechanism provides the lowest throughput. Once the video rate exceeds the $1,320 \mathrm{kbps}, \mathrm{M}-\mathrm{AVG}$ provides the best throughput.

Figure 13(b) depicts the delay of each mechanism when various video rates are used. The WCG mechanism provides the largest delay among other mechanisms. This is due to its low transmission rate, which necessitates more time to transmit a frame. On the contrary, the lowest delay is obtained with the Average mechanism.

Figure 13(c) and 13(d) illustrate the performance of all mechanisms under study in terms of BLER and FI respectively. It is worthy to notice that the conventional WCG provides the lowest BLER and the highest FI. The proposed M-WCG introduces a close result to the WCG. Moreover, the M-AVG mechanism provides a moderate performance, whereas, the Average mechanism provides the worst BLER and FI among all other mechanisms. Overall, the M-AVG mechanism performance is significantly better than the Average mechanism. However, the performance of each mechanism does not much change with all video rates. This is because the BLER and FI are directly affected by the users' channels quality. The high video rates slightly affects the BLER, because the video frame size becomes large and requires several TTIs to be transmitted. During a long frame transmission time, and due to fast fading, some users may fail to receive part of the frame, which results in dropping the frame. Thus, the probability of BLER increases as the frame size increases. 
Figure 13 Performance comparison of WCG, M-WCG, AVG and M-AVG using different video rates, (a) average user throughput (b) average delay (c) block error rate (d) FI (see online version for colours)

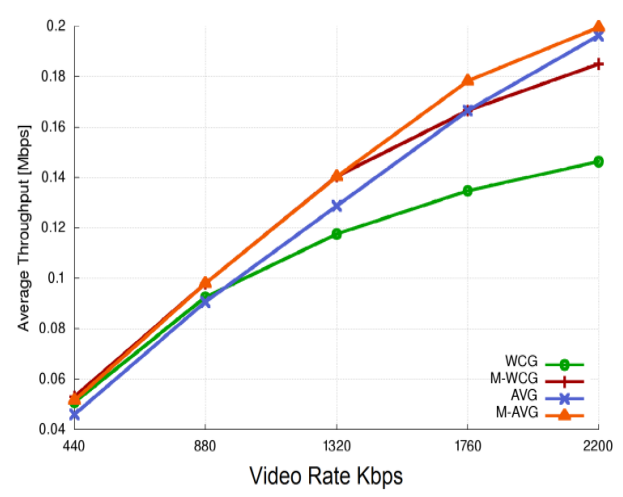

(a)

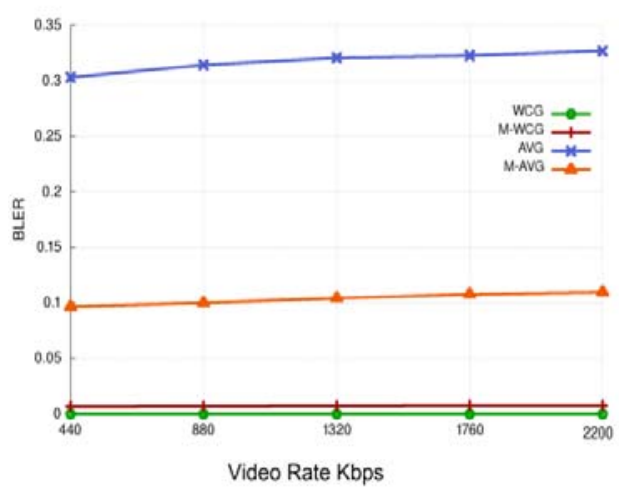

(c)

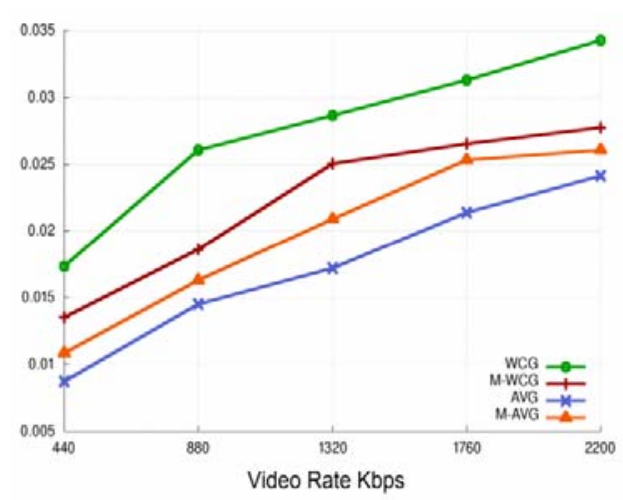

(b)

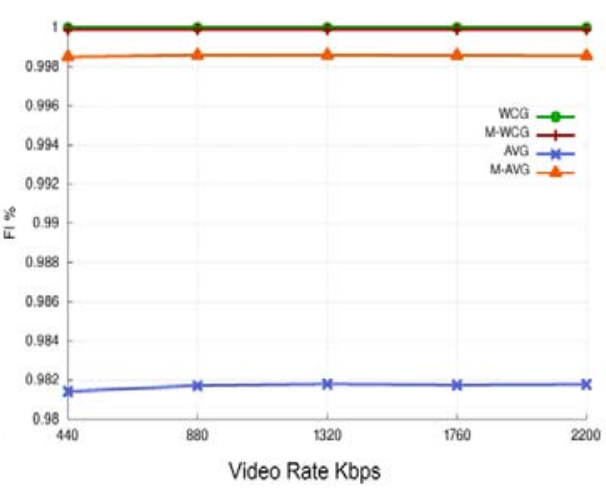

(d)

\subsection{Different number of users}

The third scenario was conducted to investigate how the number of users in a multicast group affects the performance of all mechanisms under study. As shown in Figure 14, the throughput and delay of WCG are degraded as the number of users is increased. The reason behind this is the probability of finding a cell edge user increases as the number of users increases. Other mechanisms' performance does not change when the number of users increases.

Compared to the conventional WCG and the average mechanisms, the proposed mechanisms show better performance in view of throughput BLER and FI. However, the average MCS introduces less delay than all other mechanisms at all video rates with comparable or better throughputs at high video rates. The high BLER of the average mechanism (more than 3\%) makes it unreliable to be used in a real system without combining other techniques such as opportunistic, multirate, multilayer multicast 
networks. Combining with any one of these techniques would improve the proposed mechanisms. This idea can be a future work.

Figure 14 Performance comparison of WCG, M-WCG, AVG and M-AVG using different UEs group sizes (see online version for colours)
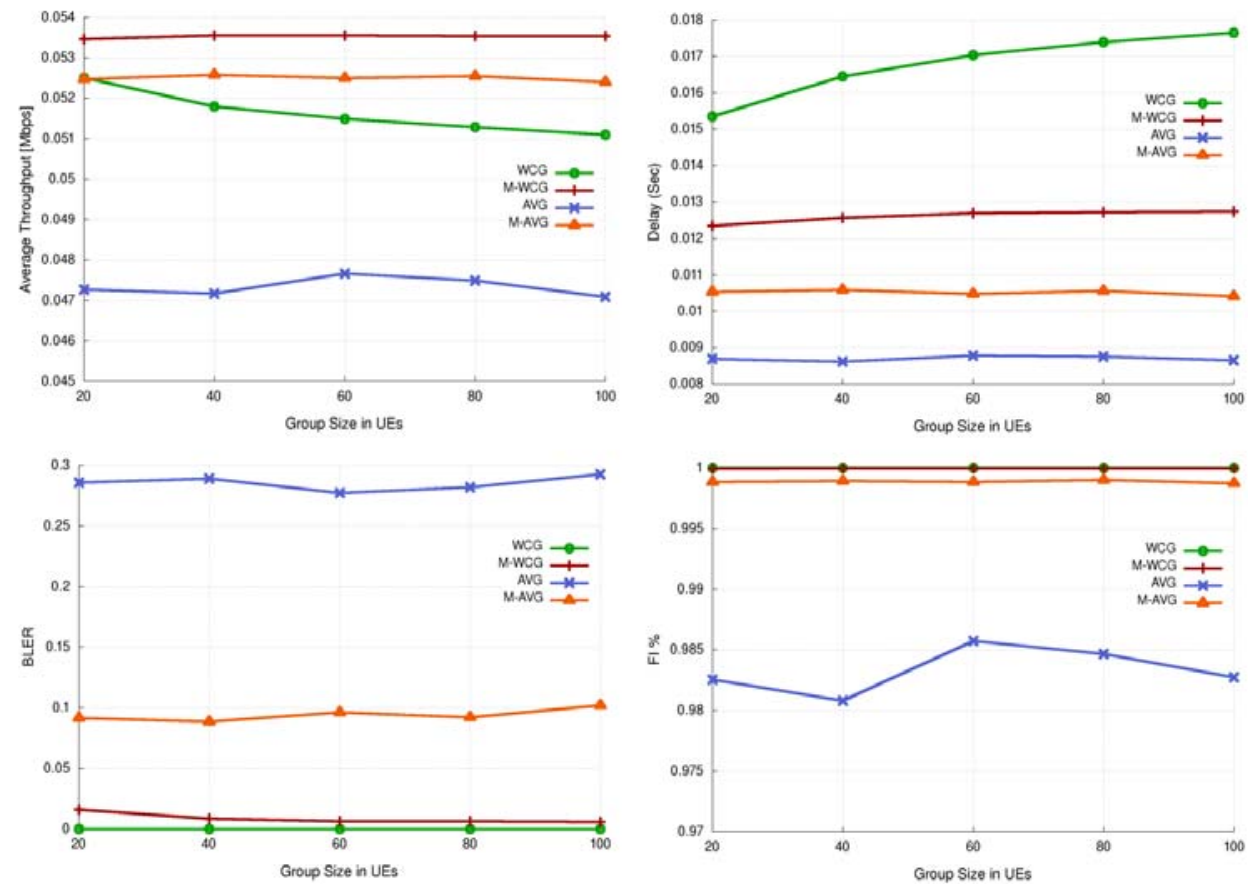

\section{Conclusions}

The increasing demand for more bandwidth for mobile broadband has created pressure on broadcast television with respect to spectrum allocation to traditional broadcast television and the mobile broadband using e-MBMS LTE.

In this paper, two mechanisms (M-WCG and M-AVG MCS level mechanism) were proposed to efficiently select the optimal MCS level based on the StD of users' MCSs level. The proposed mechanisms have been evaluated using LTE-Sim. The simulation results show that, in comparing to WCG, the M-WCG mechanism increases the performance, in term of throughput, PLR, and delay, with very little degradation in the FI; whereas, the M-AVG introduces a high FI in compare to the conventional average with degreasing in delay. Moreover, the PLR and throughput of M-AVG are stay better than as in conventional average while the number of MGs still small.

As for the next work, the use of multirate technique should be considered. Thus, the data will be transmitted on two or three different rates; each rate will be assigned to a subgroup. The data will be transmitted with the rate of the WCG in the subgroup. 


\section{References}

3GPP (2015) Technical Specification Group Services and System Aspects; Multimedia Broadcast/Multicast Service (MBMS); Architecture and Functional Description, TS 23.246 V13.3.0 (Release 13).

3rd Generation Partnership Project (3GPP) (2006) MBMS Modulation and Coding State Selection, TS R2-061985, G.T.R. WG2, June.

3rd Generation Partnership Project (3GPP) (2013) Evolved Universal Terrestrial Radio Access (EUTRA); Physical layer procedures, TS 36.213, October [online] http://www.3gpp.org/ftp/ Specs/html-info/36213.htm.

Alexiou, A., Bouras, C. and Kokkinos, V. (2010) Efficient Power Allocation in e-MBMS enabled $4 G$ Networks, Information Resources Management Association.

Alexiou, A., Bouras, C., Kokkinos, V., Papazois, A. and Tsichritzis, G. (2010) 'Spectral efficiency performance of MBSFN-enabled LTE networks', in IEEE 6th International Conference on Wireless and Mobile Computing, Networking and Communications (WiMob), pp.361-367.

Alghamdi, I., Alshehri, D.A.F., Alghamdi, A., Kerim, B. and Budiarto, R. (2016) 'Cloud-based retrieval information system using concept for multi-format data', Computer Engineering and Applications, Vol. 5, No. 1.

Anis, M.M., Lagrange, X. and Pyndiah, R. (2015) 'Cellular-based real-time flow repair for broadcast flows', IEEE Transactions on Broadcasting, September 2015, Vol. 61, No. 3, pp.457-469.

Anis, M.M., Lagrange, X. and Pyndiah, R. (2016) Overview of evolved Multimedia Broadcast Multicast Services (eMBMS), [Research Report] Dépt. Réseaux, Sécurité et Multimédia (Institut Mines-Télécom-Télécom Bretagne-UEB), p.48.

Bochrini, S., Bouras, C. and Kokkinos, V. (2013) 'Efficient MCS selection mechanisms for multicasting over LTE networks', in The 6th Joint IFIP Wireless and Mobile Networking Conference (WMNC), April, pp.1-8.

Chen, L., Wang, X. and He, G. (2012) 'Resource allocation with coding schemes for multicast services in single frequency networks', in IEEE International Conference on Communications (ICC), pp.5498-5502.

Chen, X., Yi, H., Luo, H., Yu, H. and Wang, H. (2011) 'A novel CQI calculation scheme in LTE LTE-a systems', in International Conference on Wireless Communications and Signal Processing (WCSP), November, pp.1-5.

Cohen, R., Grebla, G. and Katzir, L. (2010) 'Cross-layer hybrid FEC/ARQ reliable multicast with adaptive modulation and coding in broadband wireless networks', IEEE/ACM Transactions on Networking (TON), Vol. 18, No. 6, pp.1908-1920.

Fan, J., Yin, Q., Li, G., Peng, B. and Zhu, X. (2011) 'MCS selection for throughput improvement in downlink LTE systems', in Proceedings of 20th International Conference on Computer Communications and Networks (ICCCN), July, pp.1-5.

Fantacci, R., Marabissi, D., Tarchi, D. and Habib, I. (2009) 'Adaptive modulation and coding techniques for OFDMA systems', IEEE Transactions on Wireless Communications, September, Vol. 8, No. 9, pp.4876-4883.

Heyn, T., Morgade, J., Petersen, S., Pfaffinger, K., Lang, E., Hertlein, M. and Fischer, G. (2016) 'Integration of broadcast and broadband in LTE/5G (IMB5) - experimental results from the eMBMS testbeds', Proceedings of 2016 European Conference on Networks and Communications (EuCNC), June 2016, DOI: 10.1109/EuCNC.2016.7561055.

Ji, Y., Chen, F. and Liu, L. (2012) 'MCS selection for performance improvement in downlink TD-LTE system', in The Second International Conference on Business Computing and Global Informatization (BCGIN), October, pp.687-690.

Kim, J. and Cho, D-H. (2005) 'Enhanced adaptive modulation and coding schemes based on multiple channel reportings for wireless multicast systems', in IEEE 62nd Transactions on Vehicular Technology Conference, VTC-2005-Fall, September, Vol. 2, pp.725-729. 
Koh, C.H. and Kim, Y.Y. (2006) 'A proportional fair scheduling for multicast services in wireless cellular networks', in IEEE 64th Vehicular Technology Conference, VTC-2006, September, pp.1-5.

Lecompte, D. and Gabin, F. (2012) 'Evolved Multimedia Broadcast/Multicast Service (eMBMS) in LTE-advanced: overview and rel-11 enhancements', IEEE Communications Magazine, Vol. 50, No. 11, pp.68-74.

Liang, Y-C., Chou, C-C. and Wei, H-Y. (2011) 'Modeling and analysis of applying adaptive modulation coding in wireless multicast and broadcast systems', Wireless Networks, Vol. 17, No. 5, pp.1373-1386.

Monserrat, J.F., Calabuig, J., Fernandez-Aguilella, A. and Gomez-Barquero, D. (2012) 'Joint delivery of unicast and eMBMS services in LTE networks', IEEE Transactions on Broadcasting, Vol. 58, No. 2, pp.157-167.

Piro, G., Grieco, L.A., Boggia, G., Capozzi, F. and Camarda, P. (2011) 'Simulating LTE cellular systems: an open-source framework', IEEE Transactions on Vehicular Technology, Vol. 60, No. 2, pp.498-513.

Rong, L., Haddada, O.B. and Elayoubi, S-E. (2008) 'Analytical analysis of the coverage of a MBSFN OFDMA network', in Global Telecommunications Conference, IEEE GLOBECOM 2008, pp.1-5.

Seeling, P. and Reisslein, M. (2005) 'Evaluating multimedia networking mechanisms using video traces', IEEE Potentials, Vol. 24, No. 4, pp.21-25.

Sheng, Y., Peng, M-G. and Wang, W-B. (2008) 'A novel adaptive modulation and coding strategy based on partial feedback for enhanced MBMS network', Journal of China Universities of Posts and Telecommunications, Vol. 15, No. 1, pp.48-54.

Wang, H., Schwefel, H-P. and Toftegaard, T.S. (2008) 'History-based adaptive modulation for a downlink multicast channel in OFDMA systems', in IEEE Wireless Communications and Networking Conference, (WCNC 2008), pp.1588-1592.

Won, H., Cai, H., Eun, D.Y., Guo, K., Netravali, A., Rhee, I. and Sabnani, K. (2009) 'Multicast scheduling in cellular data networks', IEEE Transactions on Wireless Communications, Vol. 8, No. 9, pp.4540-4549.

Zhang, L., He, Z., Niu, K., Zhang, B. and Skov, P. (2009) 'Optimization of coverage and throughput in single-cell EMBMS', in IEEE 70th Vehicular Technology Conference Fall (VTC 2009-Fall), September, pp.1-5. 